\title{
Informationswirtschaft
}

Markus Nini*

\section{Wissenswert - Warum Wissen die Währung der Zukunft ist}

DOI 10.1515/iwp-2016-0050

Money makes the world go round? Nicht unbedingt! Egal ob BitCoins, Tauschbörsen, KoKonsum oder Sharing-Plattformen: Die Bedeutung von Geld als primärem Zahlungsmittel scheint langsam zu schwinden. Der Austausch von Erfahrungen, Know-how oder auch Produkten ganz ohne finanzielle Gegenleistung wird im Zuge der Sharing Economy immer populärer. Mit diesem Tauschmittel hat sich nicht nur ein ganz eigener Wirtschaftszweig entwickelt; es ist so alt wie die Menschheit selbst: das Wissen.

\section{Ein Wert mit Kopierschutz}

Wissen als einen exakten ökonomischen Wert zu erfassen, ist faktisch nicht möglich: Es gibt keine Umrechnungstabellen oder Formeln. Ein möglicher Richtwert könnte aber einerseits die Ersparnis von Zeit, materiellen und personellen Ressourcen sein, der durch effektives Wissensmanagement erreicht wird. Andererseits gibt auch der Preis, der für die Weitergabe von Wissen verlangt oder als Wettbewerbsvorteil verstanden wird, Auskunft über den „Geldwert“ von Kenntnissen und Know-how. Dieser Wert ist im Laufe des 21. Jahrhundert immer weiter gestiegen. Im Gegensatz zu anderen Tauschmitteln kann Wissen jedoch nicht einfach angeeignet oder, vor allem in Unternehmen, nicht blind übertragen werden, weil es nur in einem gewissen Kontext einen wirklichen Sinn ergibt. Wissen beinhaltet sozusagen eine Art Kopierschutz, der sich in Form von Kultur, Qualifikation und Erfahrung der Mitarbeiter auszeichnet. Wissenstausch erfordert somit, dass Wissen verstanden, übersetzt und kontextualisiert wird, weil es ansonsten nicht anschlussfähig und somit wertlos ist. Es kann als Vernetzung von Informationen verstanden werden, welche ihre Träger zu gezielten Handlungen befähigt. Durch die Digitalisierung steigt einerseits die Menge bereitgestellter Informationen, welche so an Wert ver-

*Kontaktperson: Dr. Markus Nini, Cooperational Excellence OG, Innsbrucker Bundesstraße 27, 5020 Salzburg, Österreich,

E-Mail: markus.nini@ckju.net, lieren, andererseits erhöht sich dadurch die Bedeutung von Kontextwissen.

\section{Gib weiter, was du weißt - und werde dadurch schlauer}

(Aus-)Tauschplattformen liegen momentan voll im Trend: ob klassische E-Learning-Plattformen, wie iversity, Online-Bildungseinrichtungen, wie die Kiron University, Talenttauschbörsen, wie das New Yorker Skillshare.com, oder moderierte Kollaborationsplattformen, wie ckju.net. Sie alle haben eines gemeinsam: Ausgetauscht werden nicht Waren oder Produkte gegen Geld, sondern Fähigkeiten, Talente, Know-how und Bildung. Anders als bei Vorträgen oder in Foren, erfolgt die Wissensvermittlung hier meist nicht frontal, sondern ist durch einen beidseitigen und gemeinschaftlichen Wissensaustausch gekennzeichnet. Auch Unternehmen und Organisationen profitieren von den Möglichkeiten eines unternehmensinternen und -externen Wissensaustauschs. Bereits 2008 zeigte die Metastudie des Rotterdamer Managementprofessors Raymond van Wijk, dass Wissenstransfers die Lernfähigkeit und das Innovationspotential von Unternehmen und Organisationen sichern und damit zur Wettbewerbs- und Überlebensfähigkeit beitragen.

\section{Eine gute Idee ist besser als jedes Startkapital}

Dass Wissen als Ressource immer bedeutender wird, während klassische physische und materielle Produktionsfaktoren an Relevanz verlieren, lässt sich auch anhand eines aktuellen Phänomens vermuten: Immer häufiger ist zu beobachten, dass junge Start-ups mit wenigen Mitarbeitern und geringem Kapitalvermögen weltweit aktiv sind und teilweise an der Börse höher bewertet werden als alteingesessene Unternehmen. Dabei ist nicht selten alles, was diese neuen Unternehmen an Startkapital vorweisen kön- 
nen, eine gute Idee, die sowohl Mitarbeiter als auch potentielle Kunden überzeugt. Ein weiterer Indikator für die wachsende Bedeutung von Wissen und dessen Austausch ist das steigende Angebot an Open-Innovation-Methoden und Crowdsourcing-Plattformen, in denen eine Gruppe freiwilliger User gemeinsam Projekte entwickelt und neue Ideen für Unternehmen erarbeitet - und hohe Preisgelder dafür bekommt. Allerdings gehen auch viele Teilnehmer an derartigen Wettbewerben leer aus oder können nicht überzeugen.

\section{Wissensaustausch als Erfolgsrezept}

Was mit Lean Management und agilen Methoden ursprünglich in der Softwareindustrie begonnen hat, kommt nun immer mehr in Unternehmens-, Produktions- und auch in Lernprozessen zum Einsatz: Flexible und agile Methoden, die kollaborative Arbeit unterstützen und unmittelbar auf veränderte Anforderungen reagieren können, werden immer gefragter. Mit gutem Beispiel gehen die Kommunen in Kanada voran: Im Municipal Benchmarking Network Canada tauschen sich 37 Kommunen mit Wissen und Erfahrungen regelmäßig untereinander aus. Anstatt sich nur aus der Perspektive des Wettbewerbers zu sehen, bieten Think Tanks branchenübergreifende Arbeitskreise und kooperative Benchmarking-Projekte zwischen und innerhalb von Unternehmen, wie sie etwa der Verband der Automobilindustrie eingerichtet hat, Möglichkeiten des Austauschs, der Vernetzung und Generierung von Wissen. Ähnlich arbeitet das Knowledge-Portal ShareNet im Vertrieb von Siemens ICN. Zentrales Ziel des Portals ist es, die Wiederverwendung von bereits geschaffenem, unternehmensinternem Wissen zu erhöhen und somit zu verhindern, dass mehrfach gleiches Wissen erarbeitet wird.

\section{Fazit}

Das Informationszeitalter hat sich zu einer Knowledge Sharing-Ära weiterentwickelt. Wurde früher vor allem nach Antworten und Informationen zu bestimmten Fragen gesucht oder sich in Netzwerken ausgetauscht, geht es heute mehr und mehr um die gemeinsame Entwicklung, den Austausch und die Vernetzung von Wissen. Aktuelle Methoden können identifiziert, Innovationsprozesse durch Input von außen angereichert und Arbeitsprozesse kollaborativ und ortsunabhängig durchgeführt werden. Für Unternehmen bieten Knowlegde-Sharing-Plattformen Chancen, Know-how schneller, agiler und flexibler auszutauschen und so ihre Lernfähigkeit und ihr Innovationspotential weiterzuentwickeln. Wer in Zukunft effizienter und schneller lernt, wird gewinnen.

Deskriptoren: Wissen, Technology Transfer, Innovation, Informationsnetz

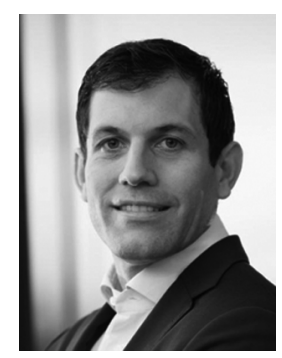

\section{Dr. Markus Nini}

Cooperational Excellence OG Innsbrucker Bundesstraße 27 5020 Salzburg

Österreich

markus.nini@ckju.net

Dr. Markus Nini ist Gründer und CEO der moderierten Beratungsund Weiterbildungsplattform ckju.net. Seine Karriere begann Nini als Unternehmensberater im Anlagen- und Maschinenbau sowie als Projektleiter in der Automobilindustrie. Es folgten Aufenthalte unter anderem in China, Brasilien und Indonesien, wo er als Operations Manager die außereuropäische Expansion eines europäischen Technologiekonzerns maßgeblich vorantrieb. Seit mehr als zehn Jahren ist er nun in der Unternehmens- und Organisationsberatung tätig, vorrangig für innovationsgetriebene Branchen wie Automotive sowie Anlagen und Maschinenbau. Markus Nini hat an der TU Wien über Teamentwicklung im Anlagenbau promoviert und studierte Organizational Behaviour am Birkbeck College, University of London. 